Historic, Archive Document

Do not assume content reflects current scientific knowledge, policies, or practices. 



\section{$\frac{4 c(0)}{h^{2}}$ \\ VICTOR JOHANSEN SEED GROWER \\ Arroyo Grande, Cal.}

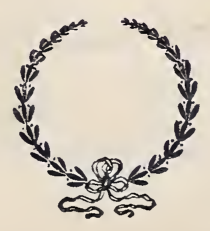




\title{
Victor Johansen
}

\section{Seed Grower}

\author{
Flower Seeds a Specialty \\ Arroyo Grande, Cal.
}

The greatest care is exercised in producing and keeping stock pure, but no warranty is given, expressed or implied.

STOCK SEED: Furnished by the Grower.

BAGS: Extra, at market value.

SHIPMENTS: As soon after harvest as practicable.

DELIVERY: F. O. B. Oceano, California.

TERMS: Three months from date of delivery on large orders.

Two months from date of delivery on very small orders.

\section{Per Cent Off for Cash}

VICTOR JOHANSEN, Arroyo Grande, Cal.:

At prices affixed, book my order for following varieties and quantities of seeds, subject to terms and conditions as above, and to pro rata delivery in the event of a short crop. 


\section{PRICE}

Alyssum

Alyssum, Sweet Alyssum - $-\$ .75$

Little Gem - - - .

\section{Aster}

Aster, Queen of the Market - -2.00

Queen of the Market,

Separate Colors - -

Semples Branching, Separate Colors

Semples Brauching, Hixed Colors

“Vicks Branching, Separate Colors

“ Tall White Branching, Comet snow White - $\quad 3.00$

"Freedom Fine Branching, Light Rose

\section{Candytuft}

Candytuft, White Rocket - - - .to

“ Empress White - - .50

" "Giant Hyacinth" White - - - .65

، Lilac and Purple - $\quad .60$

\section{Cosmos}

Cosmos, Mammoth White - $\quad .60$

" Nammoth Crimson - .60

“ Mammoth Pink - - .60

“ Early Flowering Dawn - .90

“ Early Flowering, Pink and White - - - .65

Klondyke Yellow, New - .90

\section{Celosia, Cockscomb}

Celosia, Cockscomb, Empress - 3.00

" " Japonica - 2.75

. New Crimson-feathered 3.00

\section{Carnation}

Carnation, Marouerita half dwarf double - - -

“ Margaret Double White, Pure - - - Double Large Flowering

\section{Dahlia}

Dahlia Cactus Double, Mixed Colors - - - -

“ Single, Mixed Colors - 1.75

\section{Dianthus}

Dianthus, Heddewiggii, Finest Mixture

“ The Bride - $\quad$ - 1.25

“ Eastern Queen - 1.25

Crimson Belle

\section{Escholtzia}

Escholtzia, Golden West

Alba White

Carminea

Kochia Scoparia

Kochia Scoparia Trichophilum

\section{Mignonette}

vimnonette, Victoria Crimson - \$.45

" New Ruby . - . .50

“ Allen's Defiance - .55

" Nachet - - - . 45

“ Golden Queen - - . $\quad$.

" حedorata, Large flow-

4 Hollyhock

Hollyhock, double, Red and White 3.50

Annual, New Double - 4.50

\section{Phlox Drummondi}

Phlox Drummondi Grandiflora, Separate colors - finest mixtures - $\quad-200$

Phlox, Dwarf Fireball $\quad$ - $\quad$ - 4.50

" Snowball - $\quad 4.50$

Finest Mixtures - $\quad 4.00$

\section{Poppy}

Poppy Tulip, Glaucum Scarlet - 2.00

Poppy, Shirley $\quad-\quad$ - $\quad-\quad$ - .5j

Poppy, Miss Sherwood - $\quad-.55$

Danebrog - - - - . 55

Carnation, Flowered - $\quad-\quad .50$

White Swan - $\quad-\quad 50$

Iceland, Nudicaule $\quad \therefore 3.50$

Oriental, very large, new - 3.00

\section{Salvia}

Salvia, Splendens, Scarlet

Bonfire Scarlet .

\section{Nasturtium}

Nasturtium Seed, Tall varieties . . 28

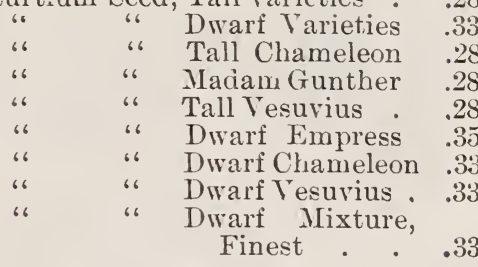

\section{Stock}

Stock, Ten Weeks German, Large Flowering, of High Doubleness 2.50

Stock, Ten Weeks German, Fine Mixture

Stock, Ten Weeks German, White Princess Alice

3135

\section{Verbena}

Verbena, Mammoth, Extra Select, Large Flower . . . 2.75

Verbena, Mammoth, Fine Mixture 2.00 " Mammoth,Separate Col'rs 2.95

\section{Zinnia}

Zinnia, Tall Double, Large Flower-

Zinnia, Tall Double, Large $\dot{\text { Flower- }}$ ing, White . $\quad$. 1.00

Zinnia, Tall Double Large Flowering, Separate Colors . 1.00 4.00 Zinnia, Dwarf, Separate Colors $\quad .1 .50$ 


\section{PRICE LIST-.-CONTINUED}

\section{SWEET PEAS}

Named Varieties:

Large Quantities . . . .10

Less than 100 pounds . . . .12

Best Large Flowering, Mixerl:

Large quantities . . . . .08

Less than 100 pounds $\quad . \quad .10$

Small Orders . • • • . 12

Good Mixtures :

Large Quantities . . . . .07

Less than 100 pounds $\quad . \quad .09$

Small Orders . . . \$.10

\section{DIFFERENT}

White-

Blanche buryee.

Dorothy Eckford.

Emily Henderson.

Wont Blanc (Early).

White Wonder.

Cream, or Light Yellow.

Hon. Mrs. E. Kenyon.

Coquette.

Sue Earl.

Queen Victuria.

Sunbeams (early).

Primrose and Light Pink.

Stella IIorse.

Iarchioness of Cholmondeley.

Golden Rose.

Light Blush

Dainty

Modesty

Duchess of sutlierland

Sensation

Light Pink.

Agnes Jolnnson

Countess of Lathanl.

Gladys Unwin

Florence Spencer

Jeanie Gordon

Lovely

Katherine Tracy

Venus

Mrs. Kuight smitl

Deeper Pink Shades.

True Countess of spencer

Janet Scott

Prima Donni

Rose and Pink Shades.

Apple Blossom

Crown Jewel

Royal Rose

Triumpli

Rose.

John Ingman

Lord Roseberry

Majestic

Prince of Wales

Mrs. Dugdale

Jeanie Gordon

hed and scarlet.

Georgie Gordon

King Fdward VII

Mars

scarlet Gem

Salopian

Prince Edward of Jork
Bush Varieties, Named:

Large Quantities, per pound .20

Less than 50 pounds : : $\quad .25$

simall Orders : : : : $\quad .30$

Countess Spencer Type : : $\quad$ : 20

New or Unnamed Varieties : 25

Bush Mixed:

Large Quantities : : : $\quad:$ : 17

Less than 50 pounds $\quad: \quad: 20$

small Orilers $\quad$ : $\quad$; $\quad$;

\section{VARIETIES}

Grange and salmon shades.

Aurora

Helen Louis

Hon. F. Beauverie

Lady Mary Currie

Niss Wilmott

Jink and White.

Blanche Ferry

Earliest of $A$ il

Extra-Early Blanche Ferry

Lavender, Iauve and Purple shades.

Admiration

Captivation

Emily Eckford

Mrs Wralter Wright

lavender.

Countess of Radno

Flora Norton

Helen Pierce

Larly Nina Balfour

Larly (iussie Hamilton

Lavender, Mauve, Picotee or Edgred.

Maid of Honor

Lottie Eckford

Blue and Purple shades.

Captain of the Blues

Countess of Cadogan

Navy Blue

Romolo Piazzani

Iaroon Shades.

Black Knight

Othello

Black Michael

striped and Variegated.

America

Gray Friar

Juanita

Senator

Ramona

Pink Friar

Mixed.

Eckford Choice Mixed

Countess Spencer, Mixed

Bush Sweet Peas.

Blanclie Ferry

Cardinal

Coccine:

Dorothy Temnant

Gray Friar

Her Majesty

Lady Mary Currie

Lottie Eckforl

Monarch

Prima Donna

Royal Rose

senator

stella Morse, Mised 